\title{
CARACTERIZAÇÃO DE MEIOS POROSOS CONSTITUÍDOS POR FOLHAS DE MANJERICÃO (Ocimum basilicum L.)
}

\author{
R. A. B. LIMA-CORRÊA, J. T. FREIRE e M. C. FERREIRA \\ Universidade Federal de São Carlos, Departamento de Engenharia Química \\ E-mail para contato: mariaf@ufscar.br
}

\begin{abstract}
RESUMO - Meios porosos constituídos por folhas de manjericão foram caracterizados visando à secagem. As propriedades físicas (dimensões, espessura, esfericidade, volume, área superficial, etc.) das folhas foram determinadas, assim como a densidade bulk dos leitos em função do tamanho e umidade das folhas para diferentes graus de compactação. A condutividade térmica efetiva e o calor específico do material foram determinados para as folhas desidratadas e pulverizadas. As metodologias utilizadas permitiram a determinação das propriedades físicoquímicas e térmicas das folhas, e a obtenção de valores reprodutíveis de densidades bulk. Os resultados permitem constatar que os meios porosos de folhas sofrem alterações estruturais ao longo da secagem, que devem ser considerados na análise de processos de desidratação.
\end{abstract}

\section{INTRODUÇÃO}

A demanda por plantas medicinais e aromáticas desidratadas cresce mundialmente, como consequência de seu amplo uso para a extração de óleos essenciais e para a produção de condimentos, fármacos e produtos de higiene e limpeza (Öztekin et al., 1999). Segundo Erbay e Icier (2010a), a secagem convectiva é ainda o método mais utilizado para a preservação de alimentos, dentre os quais se encontram as plantas aromáticas e medicinais. Para a secagem de folhas, em especial, os experimentos são normalmente conduzidos em secadores de leitos fixos, como secadores de bandejas e esteiras (Jensen et al., 2011; Calín-Sánchez et al., 2013), e utilizando camadas delgadas de material (Erbay e Icier, 2010 b). O projeto e a seleção adequada de equipamentos e condições de secagem, bem como a modelagem do processo, requer o conhecimento detalhado das características do material a ser processado. Neste sentido, é importante conhecer características físicas como a forma, o tamanho, o volume, a área superficial, a massa específica, dentre outras; além de propriedades térmicas, como o calor específico e a condutividade térmica (Mohsenin, 1970). Como as propriedades físicas podem variar em função da umidade do material, conhecer esta dependência também é necessário. Além disso, em estudos envolvendo secagem, a determinação de propriedades como a densidade bulk e a porosidade média são úteis, pois estão diretamente relacionadas à permeabilidade do meio poroso e, com exceção de alguns casos, estas propriedades dependem não somente das características das partículas, mas também da técnica utilizada para o empacotamento. Uma revisão da literatura mostra que inúmeras pesquisas têm sido conduzidas com o intuito de investigar o processo de secagem de diversas espécies de folhas e, embora a determinação de propriedades físicas (Zanoelo, 2007; Zhang et al., 2012), assim como da umidade de equilíbrio (Zanoelo, 2005; Potisate e Phoungchandang, 2010; Lima e Ferreira, 2012 a) seja relativamente usual, propriedades térmicas de folhas, bem como propriedades bulk de meios porosos constituídos por este material raramente são determinadas. De maneira geral, o que se observa nos trabalhos encontrados na 
literatura sobre secagem de folhas é que os leitos são analisados como leitos convencionais, formados por partículas com formas regulares e que não se deformam. Porém, as folhas apresentam características peculiares (tamanhos extremos, formas irregulares, texturas superficiais rugosas, alta capacidade de deformação, etc.) que exercem forte influência nas estruturas dos meios porosos formados, e, portanto, serão determinantes nos processos de secagem. Uma análise realista do processo requer, dessa forma, que as características dos meios porosos de folhas sejam estudadas e incorporadas na avaliação.

Neste contexto, o presente trabalho tem como objetivo efetuar uma caracterização detalhada de folhas de manjericão (Ocimum basilicum L.). A espécie $O$. basilicum, que apresenta folhas simples, com forma oval e margens denteadas, foi escolhida por ter características representativas desta classe de materiais e também pela ampla utilização em aplicações medicinais, perfumaria e indústrias alimentícias. Foram determinadas: (i) as propriedades físicas da folha isolada (dimensões características, massa específica e umidade inicial); (ii) as propriedades dos leitos formados por um conjunto de folhas agrupadas (densidade bulk em função do tamanho e da umidade das folhas para diferentes graus de compactação do leito); e (iii) as propriedades térmicas para o material da folha (condutividade térmica efetiva e calor específico).

\section{MATERIAIS E MÉTODOS}

\subsection{Material}

As folhas de manjericão foram adquiridas no comércio local de São Carlos/SP. Após a retirada das folhas danificadas, elas foram pré-selecionadas com o intuito de reduzir a variabilidade natural no que concerne à forma e dimensões. Na caracterização física das partículas foram utilizadas, além das folhas de manjericão inteiras, folhas cortadas em duas e em quatro partes, sendo os cortes realizados conforme mostram as fotografias da Figura 1.

(a)

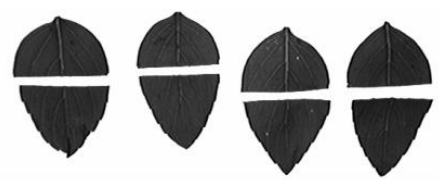

(b)

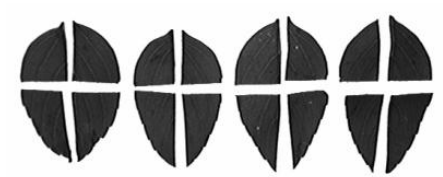

Figura 1 - Fotografias das folhas cortadas em (a) duas e (b) quatro partes.

Para a determinação das propriedades térmicas, as folhas inteiras foram secas em estufa FANEM (mod. 320-SE) na temperatura de $45^{\circ} \mathrm{C}$ por 22 horas e, em seguida, manualmente pulverizadas. A análise granulométrica do pó obtido foi realizada em uma peneira vibratória Retsch AS 200 control e o diâmetro médio de peneira $\left(D_{s}\right)$ foi calculado a partir desta análise.

\subsection{Propriedades físicas}

As folhas foram caracterizadas medindo-se a espessura média, área superficial, esfericidade, volume aparente, massa específica, umidades inicial e de equilíbrio. As dimensões características (Feret máximo e mínimo, diâmetro médio $\left(\mathrm{d}_{\mathrm{m}}\right)$ e área projetada $\left(\mathrm{A}_{\text {proj }}\right)$ ) foram medidas para trinta folhas através da técnica de análise de imagens utilizando o software Image Pro Plus 3.0. A espessura média $(\delta)$ foi obtida a partir de medidas em cinco diferentes posições da superfície, para 
amostras de trinta folhas, usando um paquímetro digital Caliper (precisão $0,01 \mathrm{~mm}$ ). A área superficial $\left(A_{p}\right)$ foi calculada somando-se a área lateral, obtida pelo produto entre o perímetro e a espessura média da folha, e o dobro da área projetada. A esfericidade $(\varphi)$ foi determinada pela relação entre a média geométrica dos três eixos perpendiculares ao corpo e o maior eixo do material (Mohnsenin, 1970). O volume aparente $\left(\mathrm{V}_{\mathrm{p}}\right)$ foi obtido por picnometria líquida com hexano e a massa específica aparente das folhas $\left(\rho_{p}\right)$ foi calculada pela relação entre a massa de folhas e o seu volume aparente. A umidade das folhas in-natura foi determinada pelo método da estufa a $(105 \pm 3)^{\circ} \mathrm{C}$ por 24 horas.

\subsection{Densidade bulk}

Para a determinação da densidade bulk $\left(\rho_{\mathrm{b}}\right)$, deve-se considerar as condições utilizadas no empacotamento do leito. A densidade bulk solta é definida como a razão entre a massa e o volume ocupado pelo sólido quando o material é inserido no recipiente sob a ação da gravidade. Quando o material é compactado por uma força normal ou por batidas sucessivas até atingir um volume constante, mede-se a densidade bulk compactada (Abdullah e Geldart, 1999). Assumindo que os leitos de folhas formam meios razoavelmente homogêneos, estas definições foram utilizadas no presente trabalho para se obter as densidades bulk solta e compactada de leitos de folhas com diferentes tamanhos (folhas inteiras, cortadas em duas e em quatro partes) e diferentes teores de umidade (in natura, totalmente seca, e com dois teores de umidade intermediários).

Aproximadamente 100,0 $\mathrm{g}$ de folhas foram utilizadas nos ensaios com as folhas in-natura. Para os demais, massas de $130,0 \mathrm{~g}$ de folhas foram secas em estufa usando $\mathrm{T}=45^{\circ} \mathrm{C}$ por tempos iguais a 2,5 h, 5,0 h e $22 \mathrm{~h}$, que forneceram amostras com teores de umidade, respectivamente, iguais a $(78,8 \pm 1,9) \%,(67,9 \pm 5,4) \%$ e $(9,6 \pm 1,9) \%$ (b.u.). Uma proveta de $2 \mathrm{~L}(8,0 \mathrm{~cm}$ de diâmetro e $46,5 \mathrm{~cm}$ de altura) foi usada para as medidas de densidade bulk e a inserção do material na proveta foi realizada com a ajuda de um funil, mantendo-se a altura de queda das folhas constante. A densidade bulk solta foi determinada inserindo-se a massa de folhas na proveta e medindo o volume ocupado pelo leito. Para as medidas de $\rho_{b}$ compactada, o volume medido na proveta foi o ocupado pela massa de folhas após a compactação do material. O uso de um dispositivo de madeira construído com uma placa horizontal localizada a aproximadamente $8,0 \mathrm{~cm}$ da base (ver Fig. 2) garantiu a padronização da altura de elevação e queda. As densidades bulk compactadas foram determinadas em função do número de batidas da proveta até que não fossem mais observadas variações no volume do leito. As densidades bulk foram, então, obtidas dividindo-se a massa adicionada pelo volume final do leito. Todas as medidas foram realizadas em triplicata.
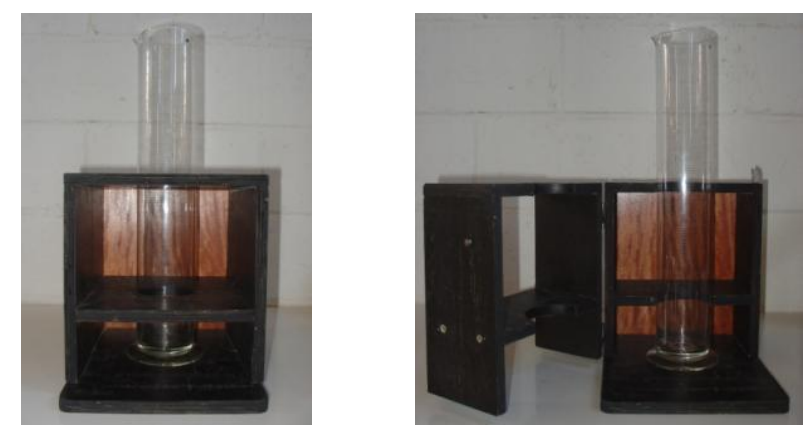

Figura 2 - Aparato experimental utilizado para as medidas de $\rho_{\mathrm{b}}$ compactada. 


\subsection{Propriedades térmicas}

A condutividade térmica efetiva $\left(\mathrm{k}_{\mathrm{ef}}\right)$ foi obtida para o material da folha com base no método da sonda linear em regime transiente, que consiste em medir a temperatura em função do tempo em um cilindro em cujo centro há um resistor linear e contínuo, que dissipa calor a baixa potência. Como leitos de folhas inteiras não são homogêneos (Lima e Ferreira, 2012 b) e não fornece bom contato térmico com a sonda de medida, a determinação de $K_{\mathrm{ef}}$ foi feita utilizando folhas desidratadas ( $\mathrm{M} \cong 12 \%$ (b.u.)) e pulverizadas. $\mathrm{O}$ aparato experimental utilizado consiste de uma célula de PVC cilíndrica (30,1 cm de diâmetro interno e 28,0 cm de altura), em cuja tampa estão afixados um termopar de chromel-alumel conectado a um leitor de temperaturas Digi-Sense ${ }^{\circledR}$ (Cole Parmer, mod. 91100-00), e uma sonda cuja resistência interna encontra-se ligada a uma fonte DC (Protek, mod. 3003B). O resistor elétrico utilizado, de especificações $600 \mathrm{~W}-220 \mathrm{~V}$, apresenta $0,64 \mathrm{~cm}$ de diâmetro e $26,5 \mathrm{~cm}$ de comprimento.

Para a realização dos experimentos (em duplicata), a célula cilíndrica foi preenchida com aproximadamente $127,0 \mathrm{~g}$ de material, as folhas secas e pulverizadas foram adicionadas em pequenas porções na célula, que foi batida manualmente depois de cada adição para a acomodação do material. A 'tampa' foi colocada e o experimento iniciado ao se aplicar uma corrente elétrica contínua de intensidade 0,11 A ao resistor. Os valores obtidos de temperatura em função do tempo foram utilizados para a construção dos gráficos de T em função de $\ln (\mathrm{t})$ e a Equação (2), resultante do truncamento da solução analítica do modelo que descreve a condução de calor em um cilindro considerado infinito, foi utilizada para a determinação da condutividade térmica efetiva. Como este modelo é válido para tempos longos, a região linear das curvas de $\mathrm{T}$ em função de $\ln (\mathrm{t})$ foi determinada utilizando-se uma rotina do software Matlab ${ }^{\circledR}$, desenvolvida por Vieira (2012), que elimina pontos experimentais de tempos iniciais até que o coeficiente de determinação $\left(\mathrm{R}^{2}\right)$ ultrapasse o valor de 0,99 . O coeficiente angular da reta ajustada foi, então, utilizado para calcular $\mathrm{o} \mathrm{k}_{\mathrm{ef}}$ do material.

$$
\frac{d T}{d \ln (t)} \cong \frac{q}{4 \pi k_{e f}}
$$

onde q é a potência térmica por unidade de comprimento (W.m ${ }^{-1}$ ) e t é o tempo (s).

\section{RESULTADOS}

\subsection{Propriedades físicas}

As propriedades físicas são mostradas na Tabela 1. As folhas estudadas apresentam baixa esfericidade, pequena espessura e volume, e elevadas dimensões lineares, como se pode observar pelos valores de Feret máximo e mínimo. A massa específica das folhas de manjericão $(0,874$ g. $\mathrm{cm}^{-3}$ ) é próxima às obtidas por Lima e Ferreira (2009) para outras espécies de folhas. A umidade inicial, $\mathrm{M}_{\mathrm{i}}$ (b.u.), das folhas in-natura, igual a $88,3 \%$, é condizente com o valor de $84,67 \%$, relatado por Özcan, Arslan e Ünver (2005) para a mesma espécie. 
Tabela 1 - Propriedades físicas das folhas de manjericão

\begin{tabular}{|c|c|c|c|}
\hline Material & Folhas inteiras & Folhas em 2 partes & Folhas em 4 partes \\
\hline Feret máximo $(\mathrm{cm})$ & $3,29 \pm 0,20$ & $2,32 \pm 0,18$ & $2,07 \pm 0,22$ \\
\hline Feret mínimo $(\mathrm{cm})$ & $1,94 \pm 0,18$ & $1,82 \pm 0,22$ & $1,11 \pm 0,11$ \\
\hline $\mathrm{d}_{\mathrm{m}}(\mathrm{cm})$ & $2,36 \pm 0,18$ & $1,87 \pm 0,16$ & $1,29 \pm 0,00$ \\
\hline $\mathrm{A}_{\text {proj }}\left(\mathrm{cm}^{2}\right)$ & $4,55 \pm 0,66$ & $2,91 \pm 0,46$ & $1,43 \pm 0,23$ \\
\hline $\mathrm{A}_{\mathrm{p}}\left(\mathrm{cm}^{2}\right)$ & 9,32 & 5,98 & 2,98 \\
\hline$\varphi(-)$ & 0,160 & 0,198 & 0,181 \\
\hline $\mathrm{V}_{\mathrm{p}}\left(\mathrm{cm}^{3}\right)$ & 0,116 & - & - \\
\hline$\delta(\mathrm{cm})$ & \multicolumn{3}{|c|}{$0,023 \pm 0,002$} \\
\hline $\mathrm{M}_{\mathrm{i}}(\mathrm{b} . \mathrm{s})$. & \multicolumn{3}{|c|}{7,10} \\
\hline$\rho_{\mathrm{p}}\left(\mathrm{g} . \mathrm{cm}^{-3}\right)$ & & $0,874 \pm 0,046$ \\
\hline
\end{tabular}

\subsection{Densidade bulk}

A Figura 3(a) mostra os gráficos de densidade bulk média em função do número de batidas para os leitos de folhas in-natura nos diferentes tamanhos estudados. É importante ressaltar que a definição de densidade média pressupõe que o material está distribuído de forma uniforme no interior do leito, mas lembramos que leitos formados por folhas são heterogêneos e os valores médios de $\rho_{\mathrm{b}}$ podem não ser representativos para todo o meio. Apesar disso, o procedimento utilizado no empacotamento resultou em valores reprodutíveis de densidade bulk média entre os diferentes ensaios, sendo as diferenças entre as réplicas inferiores a $10 \%$. Nota-se que os valores de $\rho_{\mathrm{b}}$ média para os leitos com folhas in-natura cortadas em 4 partes foram sempre superiores aos valores obtidos para os leitos com folhas inteiras e em 2 partes, indicando que uma maior compactação foi atingida nesses leitos. Observa-se ainda que, independente do tamanho das folhas, um mínimo de 70 batidas foi necessário para que os leitos atingissem um volume constante e os valores encontrados para a razão $\rho_{\mathrm{b}}$ compactada/ $\rho_{\mathrm{b}}$ solta foram bastante elevados: 2,22 para as folhas inteiras, 2,23 para as folhas cortadas em 2 partes e 2,09 para as folhas cortadas em 4 partes. Estas razões são superiores às encontradas para pós coesivos $(>1,4)$ (Abdullah e Geldart, 1999) e isto está, possivelmente, relacionado ao fato de que as folhas in-natura tendem a aglomerar devido à alta umidade e grande área de contato interfacial.

A Figura 3(b) mostra os gráficos de densidade bulk em função da umidade para as folhas inteiras e cortadas. Nota-se, de forma geral, que a densidade bulk média diminui bastante com a redução da umidade, e reduções em $\rho_{\mathrm{b}}$ solta e compactada de até $80 \%$ e $86 \%$, respectivamente, são observadas entre as folhas in-natura ( $\mathrm{M}=86 \%$ (b.u.)) e secas ( $\mathrm{M}$ entre 8 e $12 \%$ (b.u.)). A variação acentuada das densidades bulk em função do teor de umidade é um indicativo de que a estrutura de um meio poroso constituído por folhas é fortemente modificada durante um processo de secagem. É possível observar ainda que, com a diminuição de $\mathrm{M}$ (b.u.) das folhas, os valores obtidos para a razão $\rho_{b}$ compactada/ $\rho_{b}$ solta diminuem de maneira significativa. Para as folhas inteiras, esta razão diminui de 2,22 para 1,50 e para as folhas em 4 partes passam de 2,09 para 1,42. Isto ocorre porque as folhas, quando secas, perdem a capacidade de se deformar facilmente quando compactadas, e diminuem também as forças adesivas associadas à umidade, reduzindo a tendência de aglomeração observada com as folhas in-natura. Os resultados obtidos mostram claramente 
que, assim como o tamanho das folhas e o método de empacotamento do leito, a redução de umidade provoca alterações significativas nas características do meio poroso constituído por folhas. Ressalta-se que a estrutura do meio poroso está diretamente relacionada à resistência oferecida ao escoamento do ar e, consequentemente, à queda de pressão e consumo energético necessários para manter o escoamento de fluido através do leito.
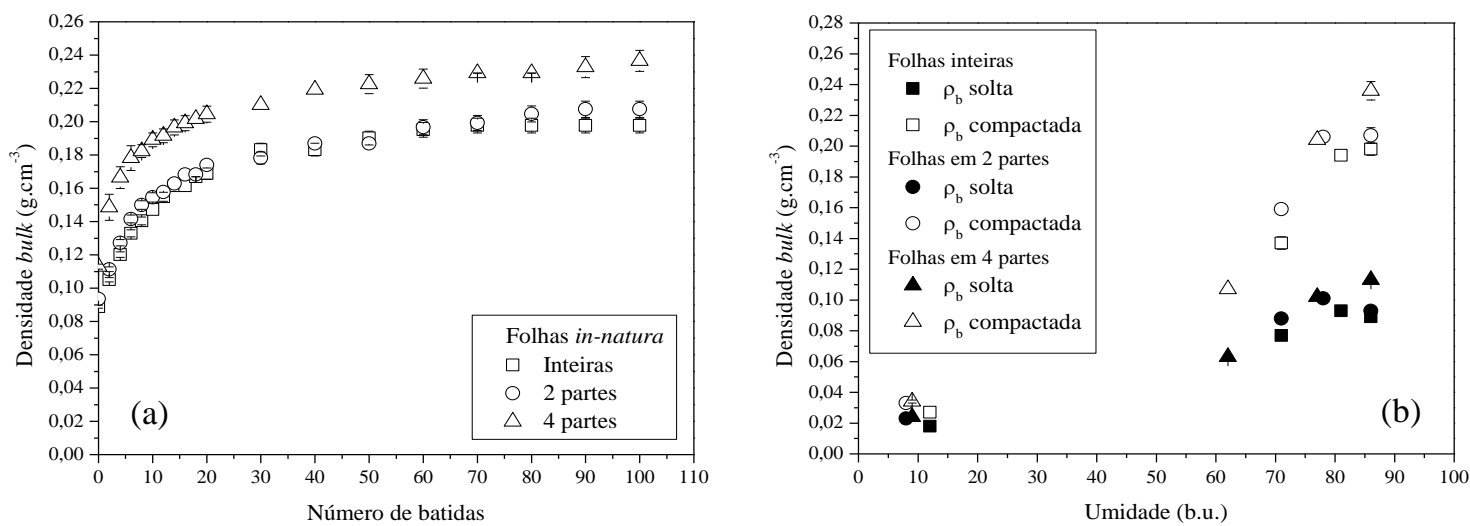

Figura 3 - $\rho_{\mathrm{b}}$ média em função (a) do $\mathrm{n}^{\circ}$ de batidas para folhas in-natura, e (b) do teor de umidade das folhas em diferentes tamanhos.

\subsection{Propriedades térmicas}

A Figura 4(a) mostra as curvas de $\mathrm{T}$ em função de $\ln (\mathrm{t})$ obtidas para as folhas de manjericão desidratadas, que resultaram em um pó com diâmetro médio de peneira $\left(D_{\mathrm{s}}\right)$ igual a $0,354 \mathrm{~mm}$. A partir do trecho linear das curvas e utilizando-se a Equação (2), determinou-se a condutividade térmica efetiva do material, cujo valor médio foi igual a $0,050 \pm 0,002 \mathrm{~W} \cdot \mathrm{m}^{-1} \cdot \mathrm{K}^{-1}$. O baixo desvio padrão e a boa reprodutibilidade observada entre os dados do ensaio e da réplica da Figura (4) confirmam a adequabilidade da sonda utilizada para determinar $\mathrm{k}_{\mathrm{ef}}$ do material. Na Figura 4(b) são mostrados os resultados provenientes da calorimetria de varredura diferencial realizada para as folhas secas e pulverizadas, onde é possível notar que o calor específico das folhas secas variou de 1,114 a $2,110 \mathrm{Jg}^{-1} .{ }^{\circ} \mathrm{C}^{-1}$ na faixa de temperatura entre 34 e $63^{\circ} \mathrm{C}$, sendo representado pelo seguinte ajuste polinomial $\left(\mathrm{R}^{2}=0,998\right)$ :

$$
C_{p}=-1,92+0,116 T-8,19 \times 10^{-4} T^{2}
$$

Zanoelo et al. (2011) determinaram o calor específico de folhas secas de erva mate (7\% b.s.) como sendo igual a $1,78 \pm 0,45 \mathrm{~J} / \mathrm{gK}$, valor este que se encontra dentro da faixa de calores específicos obtidos para as folhas secas de manjericão. 

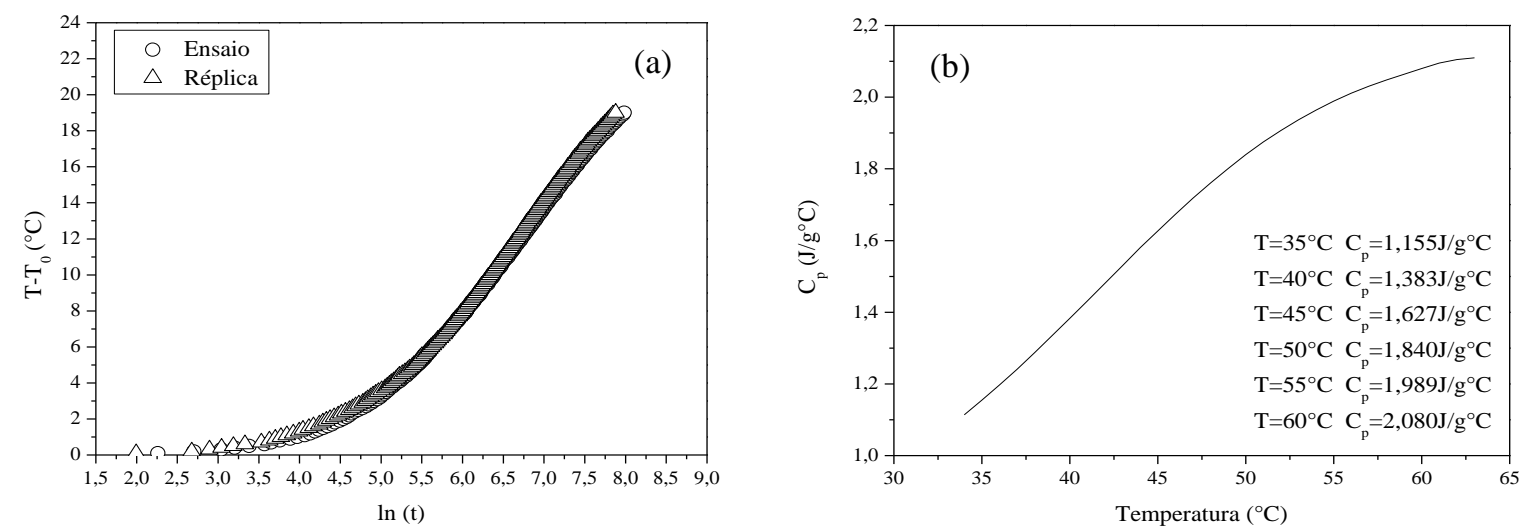

Figura 4 - Propriedades térmicas das folhas de manjericão secas e pulverizadas: (a) curvas obtidas na determinação de $\mathrm{k}_{\mathrm{ef}}$, e (b) calor específico.

\section{CONCLUSÕES}

As diferentes metodologias utilizadas na caracterização física das folhas de manjericão permitiram a determinação das principais propriedades físicas, como dimensões, áreas projetada $\left(A_{\text {proj }}=4,55 \mathrm{~cm}^{2}\right)$ e superficial $\left(A_{p}=9,32 \mathrm{~cm}^{2}\right)$, esfericidade $(\varphi=0,160)$, massa específica $\left(\rho_{p}=0,874\right.$ g. $\left.\mathrm{cm}^{-3}\right)$ e umidade inicial $\left(\mathrm{M}_{\mathrm{i}}=7,10\right.$ (b.s.)). Para o material da folha foram medidas ainda a condutividade térmica efetiva $\left(\mathrm{k}_{\mathrm{ef}}=0,05 \mathrm{~W} \cdot \mathrm{m}^{-1} \cdot \mathrm{K}^{-1}\right)$ e o calor específico. A metodologia usada para a determinação da densidade bulk dos leitos de folhas permitiu a obtenção de valores reprodutíveis. Os resultados indicaram que $\rho_{\mathrm{b}}$ foi afetada pelo tamanho das folhas, pela umidade, e também pelo grau de compactação do material no leito. Os valores variaram entre $0,089 \mathrm{~g} . \mathrm{cm}^{-3} \mathrm{e}$ $0,236 \mathrm{~g} \cdot \mathrm{cm}^{-3}$ para as folhas in natura e entre $0,018 \mathrm{~g} \cdot \mathrm{cm}^{-3}$ e $0,034 \mathrm{~g} \cdot \mathrm{cm}^{-3}$ para as folhas secas, dependendo da umidade e compactação. A densidade bulk dos leitos constituídos por folhas innatura cortadas em 4 partes foi, em média, 20\% maior do que as das folhas inteiras ou cortadas em 2 partes e as densidades bulk das folhas secas corresponderam a aproximadamente $13 \%$ dos valores obtidos para as folhas in-natura. Esta grande variação indica que durante um processo de secagem de folhas, a estrutura do meio poroso sofre fortes alterações, que precisam ser consideradas e incorporadas na análise e modelagem do processo.

\section{AGRADECIMENTOS}

Os autores agradecem à CAPES e à FAPESP (Proc. 11/07499-5) pelo apoio financeiro.

\section{REFERÊNCIAS BIBLIOGRÁFICAS}

ABDULLAH, E. C.; GELDART, D. The use of bulk density measurements and flowability indicators. Powder Technol., v. 102, p. 151-165, 1999.

CALÍN-SÁNCHEZ, Á.; FIGIEL, A.; LECH, K.; SZUMNY, A.; CARBONELL-BARRACHINA, Á. Effects of drying methods on the composition of thyme (Thymus vulgaris L.) essential oil. Drying Technol., v. 31, p. 224-235, 2013.

ERBAY, Z.; ICIER, F. A review of thin layer drying of foods: theory, modeling, and experimental results. Crit. Rev. Food Sci. Nutr., v. 50, p. 441-464, 2010 a. 
ERBAY, Z.; ICIER, F. Thin-layer drying behaviors of olive leaves (Olea europaea L.). J. Food Process Eng., v. 33, p. 287-308, 2010 b.

JENSEN, S.; MELEIRO, L. A. C.; ZANOELO, E. F. Soft-sensor model design for control of a virtual conveyor-belt dryer of mate leaves (Ilex paraguariensis). Biosystems Engineering, v. 108, p. 75-85, 2011.

LIMA, R. A. B.; FERREIRA, M. C. Avaliação de parâmetros característicos em leitos fixos e fluidizados constituídos por partículas de baixa esfericidade. In: XXXIV Congresso Brasileiro de Sistemas Particulados, 2009, Campinas, SP.

LIMA, R. A. B.; FERREIRA, M. C. Isotermas de dessorção e calor isostérico de folhas das espécies O. basilicum e S. molle. In: XIX Congresso Brasileiro de Engenharia Química, 2012 a, Vassouras, RJ.

LIMA, R. A. B.; FERREIRA, M. C. Analysis of convective drying of basil leaves. In: $18^{\text {th }}$ International Drying Symposium, 2012 b, Xiamen, China.

MOHSENIN, N. N. Physical properties of plant and animal materials. New York: Gordon and Breach Science, 1970.

ÖZCAN, M.; ARSLAN, D.; ÜNVER, A. Effect of drying methods on the mineral content of basil (Ocimum basilicum L.). J. Food Eng., v. 69, p. 375-379, 2005.

ÖZTEKIN, S.; BASÇETINÇELIK, A.; SOYSAL, Y. Crop drying programme in Turkey. Renewable Energy, v. 16, p. 789-794, 1999.

POTISATE, Y.; PHOUNGCHANDANG, S. Chlorophyll retention and drying characteristics of ivy gourd leaf (Coccinia grandis Voigt) using tray and heat pump-assisted dehumidified air drying. Drying Technol., v. 28, p. 786-797, 2010.

VIEIRA, G. N. A. Caracterização e secagem de grãos de linhaça marrom (Linum usitatissimum L.) em leito fixo e fluidizado. PPG-EQ/UFSCar, São Carlos - SP, 67p. (dissertação de mestrado).

ZANOELO, E. F. Equilibrium moisture isotherms for mate leaves. Biosystems Engineering, v. 92, p. 445-452, 2005.

ZANOELO, E. F. A theoretical and experimental study of simultaneous heat and mass transport resistances in a shallow fluidized bed dryer of mate leaves. Chem. Eng. Process., v. 46, p. 1365-1375, 2007.

ZANOELO, E. F.; BENINCÁ, C.; RIBEIRO, E. Thermophysical properties of mate leaves: experimental determination and theoretical effect of moisture content. J. Food Process Eng., v. 34, p. 2124-2136, 2011.

ZHANG, Y.; GHALY, A. E.; LI, B. Physical properties of corn residues. Am. J. Biochem. Biotechnol., v. 8, p. 44-53, 2012. 